Japan. J. Math.

Vol. 22, No. 2, 1996

\title{
Substitution invariant Beatty sequences
}

\section{By Takao Komatsu and Alfred J. van der Poorten}

(Received February 24, 1995)

\section{Introduction}

We consider sequences $\left(f_{n}\right)$, where

$$
f_{n}=[(n+1) \theta]-[n \theta], \quad n=1,2, \ldots
$$

with $\theta$ irrational and taken to satisfy $0<\theta<1$; plainly this may be assumed without loss of generality. Evidently $\left(f_{n}\right)$ is a sequence of zeros and ones. Denote by $w_{0}$ and $w_{1}$ words on the alphabet $\{0,1\}$; that is, finite strings in the letters 0 and 1 . Then the sequence $\left(f_{n}\right)$ is said to be invariant under the substitution $W$ given by

$$
W: 0 \longrightarrow w_{0}, \quad 1 \longrightarrow w_{1}
$$

if the infinite strings $f_{\theta}=f_{1} f_{2} f_{3} \cdots$ and $W\left(f_{\theta}\right)=w_{f_{1}} w_{f_{2}} w_{f_{3}} \cdots$ coincide.

This situation was discussed by Brown [2] who displays non-trivial substitutions $W$ for certain quadratic irrationals $\theta$. Subsequently Crisp, Moran, Pollington and Shiue [3] gave a complete description of all $\theta$ admitting an invariant substitution. Their result agrees with the slightly different discussion of Ito and Yasutomi [5]. Crisp et al. obtain their result from the viewpoint of cutting sequences. Berstel and Séébold [1] give a shorter but similar proof by studying the composition of two particularly simple substitutions. In this note we provide a somewhat more mechanical but efficient and rather different argument.

\section{Substitution invariance}

Set $\theta=a_{0}+\theta_{0}, a_{0}=[\theta]$, and

$$
1 / \theta_{n-1}=a_{n}+\theta_{n}, a_{n}=\left[1 / \theta_{n-1}\right] \quad n=1,2, \ldots
$$

1991 Mathematics Subject Classification. 11B83.

The work of the second author is supported in part by grants from the Australian Research Council and a research agreement with Digital Equipment Corporation. 
so that $\theta=\left[a_{0}, a_{1}, a_{2}, \ldots\right]$ is the continued fraction expansion of $\theta$. The $n$-th convergent $p_{n} / q_{n}=\left[a_{0}, a_{1}, \ldots, a_{n}\right]$ of $\theta$ is given by the recurrence relations

$$
\begin{array}{llll}
p_{n}=a_{n} p_{n-1}+p_{n-2} & (n=0,1, \ldots), & p_{-2}=0, & p_{-1}=1 \\
q_{n}=a_{n} q_{n-1}+q_{n-2} & (n=0,1, \ldots), & q_{-2}=1, & q_{-1}=0 .
\end{array}
$$

The Beatty sequence (1) has been studied and generalized by many authors. In particular, Fraenkel, Mushkin and Tassa show that

Lemma 1 ([4]). Define the sequence $\left(T_{n}\right)$ recursively by

$$
T_{0}=0, \quad T_{1}=0^{a_{1}-1} 1, \quad \text { and } \quad T_{n}=T_{n-1}^{a_{n}} T_{n-2} \text { for } n \geq 2 ;
$$

then $T_{n}$ is a word of length $q_{n}$.

If $\theta$ is irrational,

$$
f_{1} f_{2} f_{3} \cdots=\lim _{n \rightarrow \infty} T_{n} .
$$

If $\theta=p_{N} / q_{N}$ is rational, then without loss of generality one may take $N$ even, and then

$$
f_{1} f_{2} f_{3} \cdots=T_{N}^{\infty} \quad\left(=T_{N} T_{N} \cdots\right)
$$

In the light of this description of the sequence $\left(f_{n}\right)$ the following is our main result:

Theorem. Let $\theta$ be irrational, with characteristic word $f_{\theta}=\lim T_{n}$ and let $W$ be a substitution on the alphabet $\{0,1\}$. Then (i) $W\left(f_{\theta}\right)=f_{\theta}$ if and only if there is a nonnegative integer $m$ such that $W: T_{i} \longrightarrow T_{i+m}$ for each $i=1,2, \ldots$ and (ii) there is such a substitution only if $a_{i+m}=a_{i}$ for $i=3,4, \ldots$ and either $a_{2+m}=$ $a_{2}$ or $a_{1}=1$.

Proof. Plainly, if $T_{i} \longrightarrow T_{m+i}$ for all $i=1,2, \ldots$, then by Lemma 1

$$
f_{\theta}=\lim _{n \rightarrow \infty} T_{n} \longrightarrow \lim _{n \rightarrow \infty} T_{n+m}=f_{\theta} .
$$

Conversely, suppose that $W\left(f_{\theta}\right)=f_{\theta}$. We fix a sufficiently large $l$ and observe that there are $\lambda \leq \mu \leq \nu \leq \tau$ so that we may write

$$
T_{l} \longrightarrow T_{\lambda}^{c_{\lambda}} V_{\lambda}, \quad T_{l+1} \longrightarrow T_{\mu}^{c_{\mu}} V_{\mu}, \quad T_{l+2} \longrightarrow T_{\nu}^{c_{\nu}} V_{\nu}, \quad T_{l+3} \longrightarrow T_{\tau}^{c_{\tau}} V_{\tau}, \ldots
$$

Here the $c_{\lambda}, c_{\mu}, \ldots$ are positive integers and the remnants $V_{\lambda}, V_{\mu}, \ldots$ are finite, possibly empty, words too short to allow replacement of any of the parameters $\lambda, \ldots$ or $c_{\lambda}, \ldots$ by a greater one. Plainly, invariance entails that

$$
T_{l+2}=T_{l+1}^{a_{l+2}} T_{l} \longrightarrow\left(T_{\mu}^{c_{\mu}} V_{\mu}\right)^{a_{l+2}} T_{\lambda}^{c_{\lambda}} V_{\lambda}=T_{\nu}^{c_{\nu}} V_{\nu}
$$


We interrupt the argument to mention the simple but very effective

LEMMA 2. If the words $u$ and $v$ commute then there is a word $w$ and nonnegative integers $m$ and $n$ such that $u=w^{m}$ and $v=w^{n}$.

Proof. Suppose not. Let $u$ be the shortest nonempty word for which there is a counterexample $v$, and let $v$ be the shortest nonempty counterexample for that $u$. Then $u$ is no longer than $v$. Looking at the last $|v|$ symbols in $u v=v u$ we see that $v=v^{\prime} u$ for some, plainly nonempty, word $v^{\prime}$ shorter than $v$. We have $u v^{\prime} u=v^{\prime} u^{2}$, so $u v^{\prime}=v^{\prime} u$; thus, $u$ commutes with $v^{\prime}$. By hypothesis, $w$ exists such that $u=w^{m}$ and $v^{\prime}=w^{n}$. Then $v=w^{m+n}$, contradicting the hypothesis.

We are indebted to Gerry Myerson for this efficient formulation of the argument. Returning to the main argument, we note that if $\theta$ is irrational then $T_{i}$ is not periodic - that is, it is not a power of some shorter word - so, by Lemma 2, $T_{i}$ cannot commute with any nonempty shorter word, or, indeed, with any longer word that is not a power of $T_{i}$.

Suppose first that $\mu<\nu$. We note, incidentally, that this cannot lose generality by increasing $l$ if necessary, for, we cannot have $T_{\mu}(l)=T_{\nu}(l)$ for every $l$. Then $T_{\mu}^{a^{\mu+1}} T_{\mu-1}$ is a prefix of $T_{\nu}$ and thus

$$
\left(T_{\mu}^{c_{\mu}} V_{\mu}\right)^{a_{l+2}} \cdots=T_{\mu}^{a_{\mu+1}} T_{\mu-1} \cdots
$$

It follows that $V_{\mu}$ must be empty, for the apparent alternative $V_{\mu}=T_{\mu-1}$ and $a_{\mu+1}=c_{\mu}$ is excluded because then $T_{\mu}^{c_{\mu}} V_{\mu}=T_{\mu+1}$, contradicting the condition defining the remnant $V_{\mu}$.

On the other hand, suppose that $T_{\mu}=T_{\nu}$. Then

$$
\left(T_{\mu}^{c_{\mu}} V_{\mu}\right)^{a_{l+2}} T_{\lambda}^{c_{\lambda}} V_{\lambda}=T_{\mu}^{c_{\nu}} V_{\nu}
$$

and if $V_{\mu}$ is not empty then $c_{\mu}=c_{\nu}$ and, because $V_{\nu}$ is shorter than $T_{\mu}$, necessarily $a_{l+2}=1$ so $V_{\mu} T_{\lambda}^{c_{\lambda}} V_{\lambda}=V_{\nu}$. Clearly we must have $\lambda<\mu$, since $c_{\lambda}=0$ contradicts the definition of $\lambda$. Plainly then, we cannot have $T_{\nu}=T_{\tau}$, for, as just explained, that leads to $\mu<\nu$. On the other hand, $\nu<\tau$ entails $V_{\nu}$ is empty, which is absurd. Thus, in fact, always $\mu<\nu$.

The preceding argument similarly entails that both $V_{\lambda}$ and $V_{\nu}$ are empty, so it follows that

$$
T_{\mu}^{c_{\mu} a_{l+2}} T_{\lambda}^{c_{\lambda}}=T_{\nu}^{c_{\nu}}
$$

Since $\mu<\nu$, then necessarily $\lambda=\mu-1=\nu-2$ and $c_{\mu} a_{l+2}=a_{\mu+1}, c_{\lambda}=c_{\nu}=1$. Arguing similarly with $l$ replaced by $l+1$ we see that also $c_{\mu}=1$ whence $a_{l+2}=$ $a_{\mu+1}=a_{\lambda+2}$. That is, we have

$$
W: T_{l} \longrightarrow T_{\lambda}, \quad T_{l+1} \longrightarrow T_{\lambda+1}, \quad T_{l+2} \longrightarrow T_{\lambda+2}, \cdots
$$


and $a_{l+2}=a_{\lambda+2}, a_{l+3}=a_{\lambda+3}, \ldots$

Suppose however that $T_{l-1} \longrightarrow U_{\lambda-1}$. Then

$$
T_{l+1}=T_{l}^{a_{l+1}} T_{l-1} \longrightarrow T_{\lambda}^{a_{l+1}} U_{\lambda-1}=T_{\lambda}^{a_{\lambda+1}} T_{\lambda-1}
$$

Clearly $a_{l+1}>a_{\lambda+1}$ is impossible, whereas if $a_{l+1}<a_{\lambda+1}$, then we must have $\left|U_{\lambda-1}\right|>q_{\lambda}=\left|T_{\lambda}\right|$. Thus, necessarily $a_{l+1}=a_{\lambda+1}$. Hence also $T_{l-1} \longrightarrow T_{\lambda-1}$. Repeating this argument, we see that $T_{l-2} \longrightarrow T_{\lambda-2}, \ldots, T_{1} \longrightarrow T_{\lambda-l+1}$ and that $a_{l}=a_{\lambda}, \ldots, a_{3}=a_{\lambda-l+3}$.

COROLlaRY. An irrational $\theta$ admits a substitution $W$, so that $W\left(f_{\theta}\right)=f_{\theta}$, if and only if (a) $\theta=\left[0,1, a_{2}, \overline{a_{3}, \ldots, a_{n}}\right]>1 / 2$ and $a_{n} \geq a_{2}$, when the string $f_{\theta}=f_{1} f_{2} f_{3} \cdots$ is invariant under the substitution

$$
W: 0 \longrightarrow T_{n-1}^{a_{n}-a_{2}} T_{n-2}, \quad 1 \longrightarrow T_{n-1},
$$

or (b) $\theta=\left[0, a_{1}, \overline{a_{2}, \ldots, a_{n}}\right]<1 / 2$ and $a_{n}+1 \geq a_{1} \geq 2$, when the string $f_{\theta}=$ $f_{1} f_{2} f_{3} \cdots$ is invariant under the substitution

$$
W: 0 \longrightarrow T_{n-1}, \quad 1 \longrightarrow T_{n-1}^{a_{n}+1-a_{1}} T_{n-2}
$$

Proof. It is easy to check case (a) and to confirm that

$$
\begin{array}{ll}
W: T_{1}=1 & \longrightarrow T_{n-1} \\
W: T_{2}=1^{a_{2}} 0 & \longrightarrow T_{n-1}^{a_{2}} T_{n-1}^{a_{n}-a_{2}} T_{n-2}=T_{n} \\
W: T_{3}=T_{2}^{a_{3}} T_{1} & \longrightarrow T_{n}^{a_{3}} T_{n-1}=T_{n}^{a_{n+1}} T_{n-1}=T_{n+1} \\
& \vdots \\
W: T_{i} & \longrightarrow T_{n+i-2} .
\end{array}
$$

The other case is similarly straightforward.

\section{Some substitutions}

It is not too difficult to verify that the Corollary matches the result of Crisp et al. Let $R, G_{k}, H_{k}$ denote the substitutions

$$
\begin{aligned}
R: & 0 \longrightarrow 1, \quad 1 \longrightarrow 0 \\
G_{k}: & 0 \longrightarrow 1^{k} 0, \quad 1 \longrightarrow 1 \\
H_{k}: & 0 \longrightarrow 1, \quad 1 \longrightarrow 1^{k} 0
\end{aligned}
$$

To verify the correspondence between our present results and those of [3] we need just the following 
Proposition. If $\theta>1 / 2$, then

$$
\begin{aligned}
& H_{a_{2}} \circ H_{a_{3}} \circ \cdots \circ H_{a_{n-1}} \circ G_{a_{n}-a_{2}}(1)=T_{n-1}, \\
& H_{a_{2}} \circ H_{a_{3}} \circ \cdots \circ H_{a_{n-1}} \circ G_{a_{n}-a_{2}}(0)=T_{n-1}^{a_{n}-a_{2}} T_{n-2},
\end{aligned}
$$

where $a_{n} \geq a_{2}$; whereas if $\theta<1 / 2$,

$$
\begin{aligned}
& R \circ H_{a_{1}-1} \circ H_{a_{2}} \circ \cdots \circ H_{a_{n-1}} \circ G_{a_{n}+1-a_{1}} \circ R(0)=T_{n-1}, \\
& R \circ H_{a_{1}-1} \circ H_{a_{2}} \circ \cdots \circ H_{a_{n-1}} \circ G_{a_{n}+1-a_{1}} \circ R(1)=T_{n-1}^{a_{n}+1-a_{1}} T_{n-2},
\end{aligned}
$$

where $a_{n}+1 \geq a_{1} \geq 2$.

Proof. We shall check only the first relation. The others are confirmed in much the same way.

Since $G_{a_{n}-a_{2}}(1)=1$, it is sufficient to show that

$$
H_{a_{2}} \circ H_{a_{3}} \circ \cdots \circ H_{a_{n-1}}(1)=T_{n-1} .
$$

When $n=3,1^{a_{2}} 0=T_{2}$. When $n=4,\left(1^{a_{2}} 0\right)^{a_{3}} 1=T_{2}^{a_{3}} T_{1}=T_{3}$.

If $H_{a_{2}} \circ H_{a_{3}} \circ \cdots \circ H_{a_{n-3}}(1)=T_{n-3}$ and $H_{a_{2}} \circ H_{a_{3}} \circ \cdots \circ H_{a_{n-2}}(1)=T_{n-2}$, then

$$
\begin{aligned}
H_{a_{2}} \circ H_{a_{3}} \circ \cdots \circ H_{a_{n-1}}(1) & =H_{a_{2}} \circ H_{a_{3}} \circ \cdots \circ H_{a_{n-2}}\left(1^{a_{n-1}} 0\right) \\
& =T_{n-2}^{a_{n-1}} H_{a_{2}} \circ H_{a_{3}} \circ \cdots \circ H_{a_{n-3}}(1) \\
& =T_{n-2}^{a_{n-1}} T_{n-3}=T_{n-1} .
\end{aligned}
$$

On the other hand, Brown [2] uses the substitution

$$
h_{k}: 0 \longrightarrow 0^{k-1} 1, \quad 1 \longrightarrow 0^{k-1} 10 .
$$

Then we have

$$
\begin{aligned}
& h_{a_{1}} \circ \cdots \circ h_{a_{n}}(0)=T_{n}, \\
& h_{a_{1}} \circ \cdots \circ h_{a_{n}}(1)=T_{n} T_{n-1} .
\end{aligned}
$$

Thus, it is clear that $f_{1} f_{2} f_{3} \cdots$ is invariant under the substitution $k_{a_{1}} \circ \cdots \circ h_{a_{n}}$ when $\theta=\left[0, \overline{a_{1}, \ldots, a_{n}}\right]$ (Theorem $1[2]$ ). He also considers the block-to-block substitution

$$
s \longrightarrow C_{1}, \quad t \longrightarrow C_{2},
$$

where $s$ and $t$ are finite strings of 0 's and 1's and $C_{1}$ and $C_{2}$ are finite strings of $s$ 's and $t$ 's. If we allow this kind of substitution, we can leave $f_{1} f_{2} f_{3} \cdots$ with

$$
\theta=\left[0, a_{1}, \ldots, a_{l}, \overline{a_{l+1}, \ldots, a_{l+m}}\right]
$$


invariant under the substitution

$$
T_{l} \longrightarrow T_{l+m}, \quad T_{l} T_{l-1} \longrightarrow T_{l+m} T_{l+m-1}
$$

(See [2], Theorem 2). Indeed, $T_{n} \longrightarrow T_{n+m}$ holds for any integer $n \geq 1$.

Ito and Yasutomi deal with similar cases to Brown's, but their definition of the Beatty sequence includes the term $f_{0}$, and that makes a considerable difference in the present context. Since $\theta_{2 n+1}=0 T_{2 n} 0^{-1}$ and $\theta_{2 n+2}=0 T_{2 n}^{-1} T_{2 n+1} T_{2 n} 0^{-1}$ for any non-negative integer $n$ (see [5], p.299 concerning this notation), it immediately follows that $f_{0} f_{1} f_{2} f_{3} \cdots$ is invariant under the subsitution

$$
\gamma_{a_{1}, a_{2}} \circ \cdots \circ \gamma_{a_{2 n-1}, a_{2 n}}: 0 \longrightarrow 0 T_{2 n} 0^{-1}, \quad 1 \longrightarrow 0 T_{2 n-1} T_{2 n} 0^{-1}
$$

when $\theta=\left[0, \overline{a_{1}, a_{2}, \ldots, a_{2 n-1}, a_{2 n}}\right]$. In fact, for any positive integer $i$ we have $T_{i} \longrightarrow 0 T_{2 n}^{-1} T_{2 n+i} T_{2 n} 0^{-1}$ but $0 T_{i} \longrightarrow 0 T_{2 n+i} T_{2 n} 0^{-1}$.

\title{
References
}

[ 1 ] J. Berstel and P. Séébold, A characterization of Sturmian morphisms, Mathematical Foundations of Computer Science, 1993 (Gdańsk, 1993), 281-290, Lecture Notes in Comp. Sci., 711, Springer, Berlin, 1993.

[ 2 ] T.C. Brown, A characterization of the quadratic irrationals, Canad. Math. Bull., 34 (1991), 36-41.

[ 3 ] D. Crisp, W. Moran, A. Pollington and P. Shiue, Substitution invariant cutting sequences, J. Théorie des Nombres de Bordeaux, 5 (1993), 123-137.

[ 4 ] A.S. Fraenkel, M. Mushkin and U. Tassa, Determination of $[n \theta]$ by its sequence of differences, Canad. Math. Bull., 21 (1978), 441-446.

[ 5 ] Sh. Ito and S. Yasutomi, On continued fractions, substitutions and characteristic sequences $[n x+y]-[(n-1) x+y]$, Japan. J. Math., 16 (1990), 287-306.

\author{
School of Mathematics, Physics, \\ COMPUTING AND ELECTRONICS \\ MACQUARIE UNIVERSITY NSW 2109 \\ Australia \\ alf@mpce.mq.edu.au
}

